\title{
Preeclampsia y enfermedad cardiovascular: un enfoque integral para detectar las fases subclínicas de complicaciones obstétricas y cardiovasculares
}

\author{
Gloria Valdés \\ Departamento de Nefrología, Facultad de Medicina, \\ Pontificia Universidad Católica de Chile.
}

Financiamiento: Ninguno.

Antecedentes: Desde 1995 hasta la fecha la asociación entre patologías derivadas los embarazos hipertensivos y las enfermedades cardiovasculares ha generado un gran volumen de potentes evidencias epidemiológicas y clínicas.

Objetivos: Los propósitos de esta revisión son varios. Mostrar la consistencia y magnitud de la evidencia científica. Integrar los riesgos/enfermedades cardiovasculares y los problemas obstétricos a través de la disfunción endotelial. Preconizar el seguimiento postparto de la hipertensa embarazada, como una ventana de oportunidad para beneficiar la salud de las mujeres y sus hijos. Incluir la historia obstétrica como factor de riesgo de enfermedad coronaria. Proponer cuestionarios adaptables a las prácticas locales para facilitar la pronta incorporación de los índices de riesgo obstétrico y cardiovascular en dos etapas de la vida de una mujer.

Conclusión: Ha llegado el momento para que los equipos obstétricos, cardiológicos y pacientes jueguen un rol en la prevención de los riesgos y enfermedades cardiovasculares.

Palabras claves: Embarazo hipertensivo, preeclampsia, enfermedad cardiovascular, salud femenina. 


\section{Pregnancy and cardiovascular disease: a comprehensive approach to detect subclinical phases of obstetric and cardiovascular complications}

Background: From 1995 onwards the association between hypertensive pregnancies and cardiovascular disease has generated a great volume of epidemiologic and clinical evidence.

Objectives: The purposes of this review are several. To demonstrate the consistence and weight of the scientific evidence. To integrate cardiovascular risks/ diseases and obstetric complications through the link of endothelial dysfunction. To advocate postpartum follow-up after a hypertensive pregnancy as a window of opportunity to benefit the health of mothers and offsprings. To include the obstetrical history as a risk factor for coronary disease. To propose questionnaires adaptable to local practices to incorporate cardiovascular and obstetrical indexes into two stages of a woman's lifetime.

Conclusion: The time has come for obstetrical teams, cardiologists and patients to play a preventive role regarding cardiovascular risks and diseases.

Keywords: hypertensive pregnancy; preeclampsia; cardiovascular disease; women's health. 


\section{Introducción:}

La mortalidad materna en Chile entre 1990 y el 2000 descendió desde 52.1 a 23.6 por 100.000 nacidos vivos ${ }^{1}$. Al prolongar este período hasta el 2004 se observó un decremento leve a 18.5 por 100.000 nacidos vivos, que permanece relativamente estable en el $2015^{1,2}$. Como se aprecia en la Tabla 1 la mortalidad materna en nuestro país es de las más bajas en América del Sur ${ }^{1}$. Este resultado, producto de la prioridad de los programas materno-infantiles, nos obliga a seguir progresando, especialmente cuando enfrentamos una transición epidemiológica que obliga a resolver simultáneamente patologías sintomáticas y a incorporar medidas preventivas que las reduzcan en el mediano y largo plazo.

\begin{tabular}{|c|c|c|}
\hline País & $\begin{array}{l}\text { Mortalidad } \\
\text { materna } \\
\text { x } 100000 \mathrm{nv}\end{array}$ & $\begin{array}{r}\text { Mortalidad } \\
\text { neonatal } \\
\times 1000 \mathrm{nv}\end{array}$ \\
\hline Islandia & 0.8 & 0.9 \\
\hline Noruega & 3.8 & 1.3 \\
\hline Singapur & 5.0 & 1.0 \\
\hline Australia & 5.5 & 2.0 \\
\hline España & 5.6 & 1.6 \\
\hline Israel & 5.8 & 1.8 \\
\hline Japón & 6.4 & 1.1 \\
\hline Francia & 7.8 & 1.3 \\
\hline Inglaterra & 8.8 & 2.6 \\
\hline Alemania & 9.0 & 1.8 \\
\hline China & 17.7 & 6.1 \\
\hline Rusia & 18.7 & 4.4 \\
\hline Tailandia & 20.0 & 3.3 \\
\hline Chile & 20.5 & 3.9 \\
\hline Uruguay & 21.3 & 4.9 \\
\hline Estados Unidos & 26.4 & 3.3 \\
\hline México & 48.2 & 8.3 \\
\hline Brasil & 65.4 & 9.4 \\
\hline Paraguay & 100.6 & 10.0 \\
\hline Sudafrica & 159.7 & 18.2 \\
\hline India & 247.6 & 29.1 \\
\hline Pakistan & 348.6 & 37.9 \\
\hline Chad & 549.9 & 36.1 \\
\hline Guinea & 604.9 & 35.2 \\
\hline Mortalidad Global & 196.0 & 18.7 \\
\hline
\end{tabular}

En relación a las complicaciones obstétricas, es indispensable considerar el campo abierto hace más de dos déca- das por la asociación entre las enfermedades cardiovasculares con distintas formas de síndromes hipertensivos del embarazo (SHE) ${ }^{3}$ : a) hipertensión crónica, b) hipertensión gestacional, sin proteinuria ni anormalidades de laboratorio c) preeclampsia (PE) aislada o sobreagregada a una hipertensión crónica, y d) hipertensión crónica enmascarada por presiones mayores fuera de la consulta ${ }^{4}$. Ya que en la Encuesta Nacional de Salud (ENS) 2009$2010^{5}$ un 62.5 y $46.8 \%$ de los grupos de 15 a 24 años, y 25 a 44 años, respectivamente, no habían detectado ser hipertensos, es probable que la vasodilatación de la gestación temprana enmascare una hipertensión pregestacional.

Evidencias de la interconexión entre el embarazo hipertensivo y el riesgo cardiovascular remoto de la madre.

Desde las primeras comunicaciones que asociaron la PE y otros SHE con las enfermedades cardiovasculares (ECV) a mediados de los 90, numerosas publicaciones han corroborado este hecho y agregado asociaciones con aborto recurrente, desnutrición intrauterina, parto prematuro, desprendimiento de placenta y mortinatos ${ }^{6-20}$. El riesgo cardiovascular (RCV) se correlaciona con la severidad clínica de las manifestaciones maternas y fetales, como se observó para PE recurrente ${ }^{21}$ y en un registro prospectivo de una cohorte en que el riesgo de eventos coronarios aumentó 2.1 veces con una PE previa, 3.3 con desnutrición intrauterina y 5.4 con parto prematuro ${ }^{20}$. La calcificación coronaria, que antecede a la enfermedad coronaria, aumenta 3 décadas después de una $\mathrm{PE}^{22}$. $\mathrm{Mu}$ jeres sometidas a angiografía coronaria por sospecha de lesiones obstructivas presentaron manifestaciones clínicas más precoces y aumento del número de arterias estenóticas si habían presentado $\mathrm{SHE}^{23}$.

Un subanálisis de la ENS 2009-2010 mostró que las mujeres que habían presentado SHE tenían un riesgo relativo ajustado de 2.1 de ser hipertensas (Paula Margozzini, comunicación personal). En la última década, 4 revisiones sistemáticas y meta-análisis han comprobado la asociación de PE con ECV y han mostrado una gran consistencia en la magnitud del riesgo futuro; se triplica el riesgo de hipertensión arterial y se duplica el de enfermedad coronaria y de muerte cardiovascular (Tabla 2) ${ }^{24-}$ 27. A pesar de esto el tamizaje de los factores de RCV no se ha incorporado a la evaluación preconcepcional o a los primeros controles del embarazo y el seguimiento postparto de mujeres con SHE solo se efectúa en escasas instituciones ${ }^{28,29}$.

Tampoco se evalúa la historia obstétrica en mujeres que 


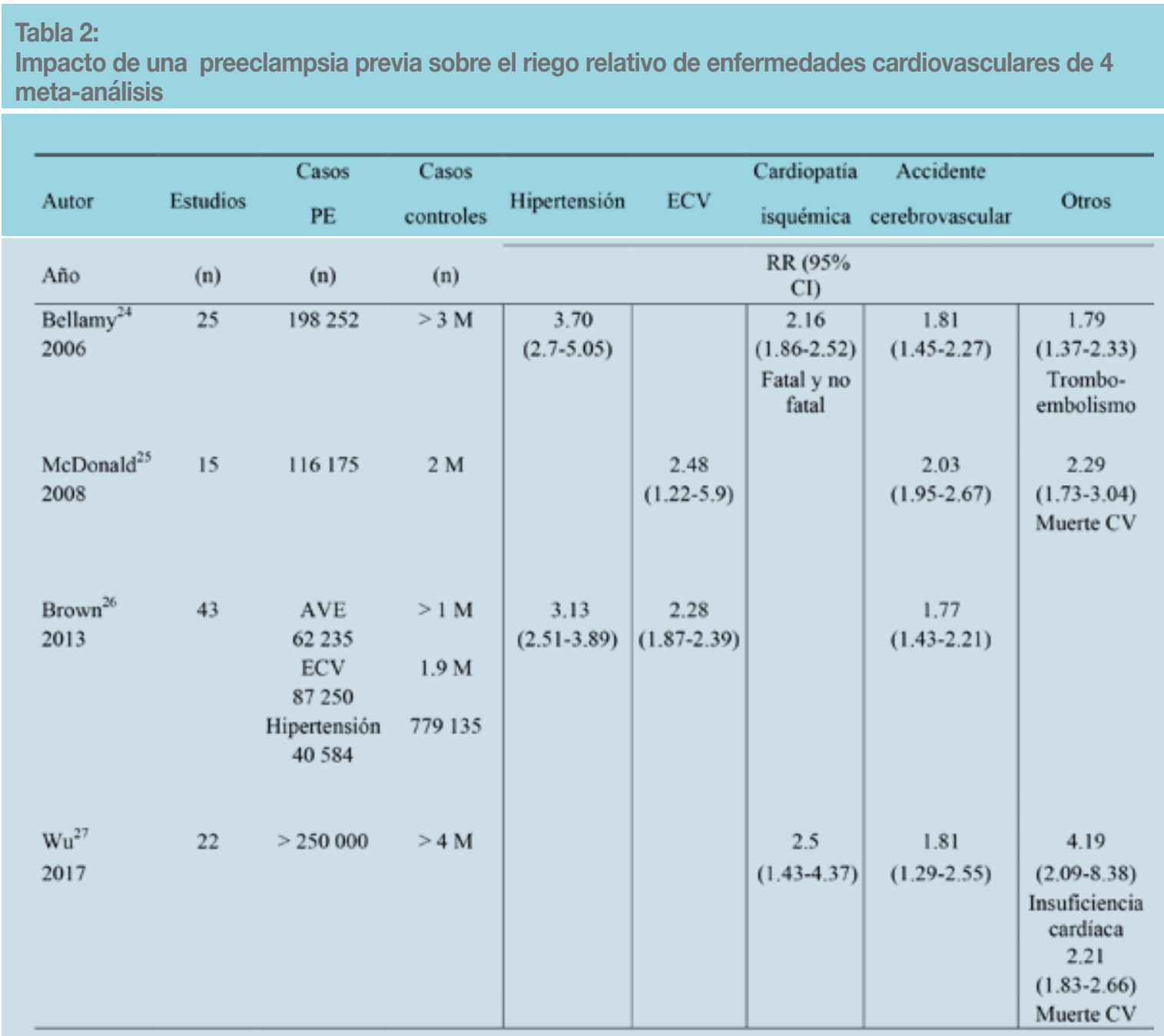

$\mathrm{M}=$ millones; $\mathrm{AVE}=$ accidente vascular encefálico; $\mathrm{ECV}=$ enfermedad cardiovascular; $\mathrm{CV}=$ cardiovascular.

consultan por síntomas de enfermedad coronaria décadas después, si bien la Sociedad Internacional para el Estudio de la Hipertensión en el Embarazo, las Asociaciones Americanas de Cardiología y de Accidente Cerebrovascular, y la Sociedad Europea de Cardiología, han enfatizado el riesgo remoto de las complicaciones gestacionales ${ }^{4,30-32}$. Esto es especialmente importante pues la isquemia miocárdica, la mayor causa de muerte en mujeres, permanece subdiagnosticada y tratada debido a varios factores ${ }^{33}$. Entre estos destacan la presentación solapada de la enfermedad coronaria en mujeres, la mantención de criterios bioquímicos y electrocardiográficos aplicados a los hombres, la menor frecuencia de lesiones coronarias y la disfunción microvascular de un $30 \%$ de las pacientes que en ausencia de lesiones obstructivas presenta más complicaciones adversas ${ }^{33-35}$. La instalación de programas preventivos postparto en las madres que fallaron en una "prueba de esfuerzo cardiovascular y metabólica" de la gestación reduciría la mortalidad cardiovascular prematura, que en Chile en el 2010 ocupó el primer lugar de las muertes prematuras, con una tasa de $57.8 \times 100.000$ habitantes $^{35}$.

Evidencias de la interconexión entre el embarazo hipertensivo y el riesgo cardiovascular temprano y remoto de su hijo. Los hijos de embarazadas hipertensas presentan mayor morbimortalidad neonatal por prematurez (espontánea o por indicación de interrupción 
por riesgo materno y/o malas condiciones fetales) y por restricción de crecimiento. Barker, en 1989, relacionó el desarrollo fetal con el aumento de presiones arteriales desde la infancia y mayor mortalidad cardiovascular en la vida adulta, abriendo el área del "Developmental Origins of Health and Disease" (DOHaD) ${ }^{37-39}$. Diferentes grupos de investigación en diversas poblaciones han demostrado que los recién nacidos con desnutrición intrauterina y/o prematurez tienen tempranamente factores de riesgo cardiovascular ( $\mathrm{RCV}$ ) genéticos, epigenéticos y ambientales; entre ellos mayores niveles de presión arterial, hiperlipidemia, resistencia a la insulina y reducción de nefrones $40-45$.

Estos hallazgos enfatizan la necesidad de convertir el embarazo y la infancia en una oportunidad privilegiada de prevención. En Chile, González et al, mostraron que la mortalidad neonatal entre 1990 y 2004 descendió de 9.0 a 5.7 por 1000 nacimientos. A este descenso se agregó la reducción de la mortalidad en el primer año de vida, y la infantil ( 7.8 a 3.1 y 3.1 a 1.7 , respectivamente $)^{2}$, revelando que a la mejor atención del embarazo y parto se agregan las políticas de salud y factores socioeconómicos. La Tabla 1 muestra que la tasa de mortalidad neonatal en el 2015 es 3.9 , que es 5 veces menor que la global ${ }^{46}$. Sin embargo, la importancia del desarrollo fetal y las tasas de mortalidad neonatal de los países que encabezan el listado demuestran que existe amplio margen para optimizar este índice y la mortinatalidad de 8.5 x 1000 nacidos vi$\operatorname{vos}^{47}$. González et al, sugieren que deben invertirse más recursos en el período que rodea el nacimiento ${ }^{2}$. Diez años más tarde es indispensable acompañar los recursos con una translación de la investigación biomédica a las atenciones obstétricas y pediátricas.

\section{Vías fisiopatológicas que unen las complicaciones ges- tacionales y la enfermedad cardiovascular.}

Maternas: Muchos RCV son comunes a ambas condiciones: hipertensión arterial, obesidad, resistencia a insulina, diabetes, hiperlipidemia, ECV familiar prematura, stress oxidativo, inflamación (autoinmune o infecciosa), apnea de sueño, condiciones procoagulantes y genotipos relacionados a $\mathrm{ECV}^{48-52}$. Otros factores comunes son el efecto preventivo leve a moderado de aspirina ${ }^{53-55} \mathrm{y}$ la semejanza entre las placas de aterosis y las ateromatosas $^{56}$. Escasas condiciones difieren entre PE y ECV, como la adolescencia con su deficiente precondicionamiento endometrial ${ }^{57}$, los embarazos múltiples con el aumento de masa placentaria y el tabaquismo que reduce el receptor soluble (sFLT-1) del factor de crecimiento vascular (vascular growth factor o VEGF) ${ }^{58}$.

El endotelio normal responde al roce tangencial del flujo sanguíneo (shear stress) y protege a lo largo de la vida de la ateroesclerosis y sus complicaciones clínicas a través de la síntesis y liberación óxido nítrico, y de factores vasoactivos relacionados que actúan sobre el lumen vascular previniendo la agregación de plaquetas, sobre la íntima reduciendo la infiltración de monocitos y la acumulación de lípidos y sobre la media relajando el músculo liso, previniendo su proliferación y la fibrosis intersticial $^{59,60}$. En presencia de un endotelio reactivo la remodelación de las arterias espirales uterinas está precondicionada por el trofoblasto extravelloso que las aproxima liberando factores vasoactivos, la transforma antes de invadirla (priming), luego remplaza su capa muscular, alcanza el lumen endotelial y las transforma en vasos saculares que proveen una perfusión placentaria adecua$\mathrm{da}^{61-62}$. El índice de pulsatilidad de las arterias uterinas a las 23 semanas refleja una remodelación adecuada o deficiente de las arterias espirales y supera la sensibilidad de los marcadores de stress oxidativo y de disfunción endotelial para predecir $\mathrm{PE}^{63}$.

Hemos postulado que un endotelio disfuncional perturba la fina sincronización entre células maternas y el trofoblasto en la $\mathrm{PE}^{64}$, causando isquemia placentaria y aumento de deportación de microvellosidades y factores solubles a la circulación materna, que acentúan la disfunción endotelial por RCV preexistentes y el desbalance entre factores vasodilatadores/vasoconstrictores y pro/ antiangiogénicos ${ }^{65-72}$. Fallas en la adaptación sistémica materna a la expansión de volumen y del débito cardíaco, elevan las cifras tensionales y exponen el defecto que décadas más tarde contribuye a las ECV ${ }^{73,74}$.

La disfunción endotelial, caracterizada por una reducción de la vasodilatación mediada por flujo, puede preceder a la PE y persiste hasta 20 años después de terminado el embarazo ${ }^{75-80}$. La disfunción endotelial se considera una secuela de la injuria provocada por los factores placentarios a la circulación materna; sin embargo, la disfunción endotelial posterior a abortos recurrentes apoya un rol causal, ya que la exposición a productos placentarios ha sido mínima e intermitente ${ }^{63}$. Esta hipótesis se refuerza por RCV asociados a un SHE o PE pre o postconcepcional (diabetes familiar, diabetes pre- o intragestacional, infartos prematuros en familiares directos, sobrepeso, obesidad, hipertensión arterial, aumento de leucocitos y triglicéridos $)^{75-81}$. También por el grosor aumentado de la íntima-media carotidea - índice preclínico de ateroesclerosis posterior a la disfunción endotelial - observado 


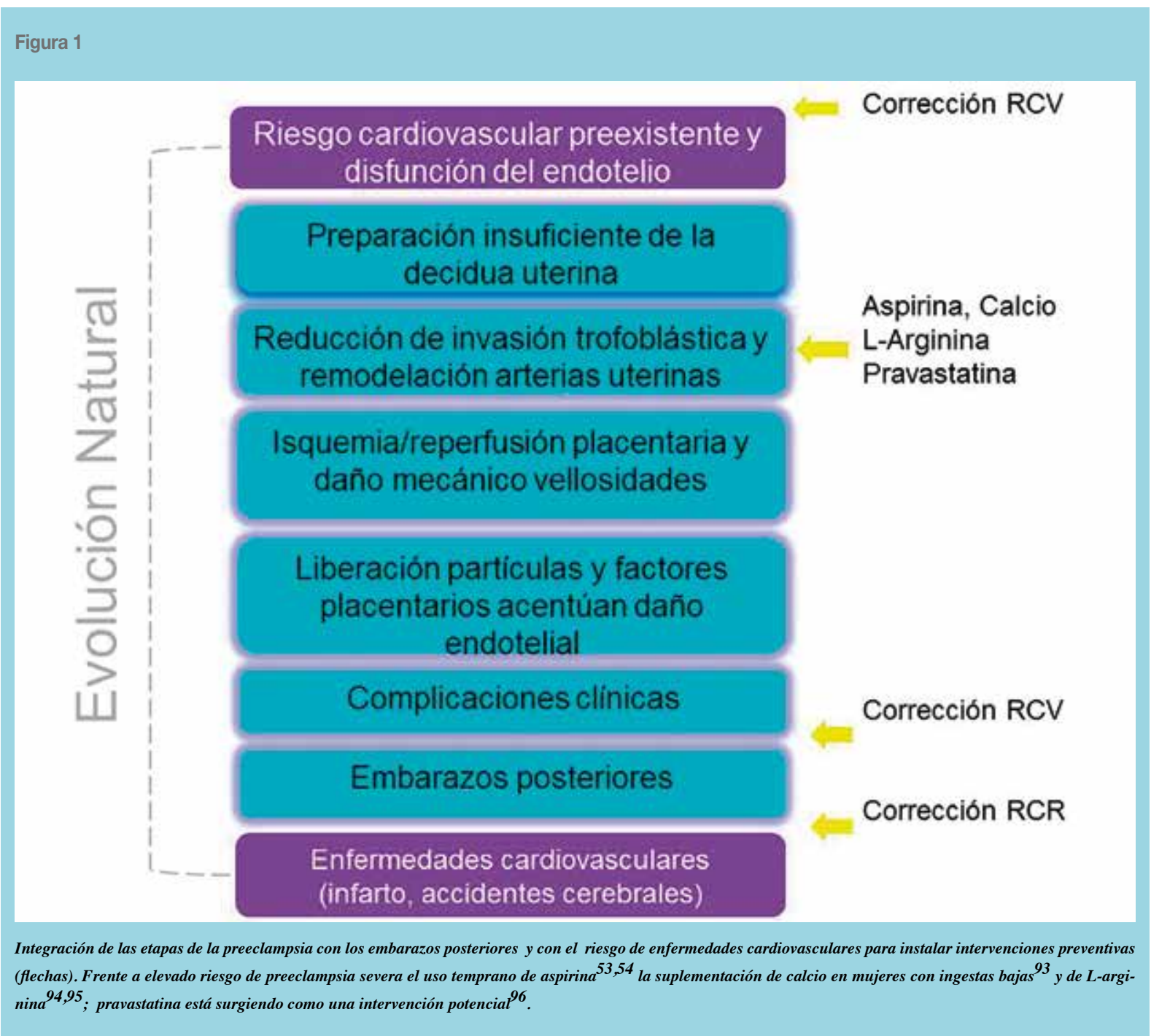

durante la PE y en la década siguiente, ${ }^{82}$.

La disfunción endotelial se convirtió en el factor central de la PE cuando Roberts, Taylor et al. ${ }^{64}$ y Redman et al. ${ }^{65}$, demostraron que la PE tiene dos etapas, una remodelación deficiente de las arterias espirales que lleva a perfusión placentaria reducida seguida del síndrome clínico provocado por injuria endotelial por factores antiangiogénicos, citotóxicos, inflamatorios, oxidativos e inmunológicos ${ }^{64-67}$. Posteriormente se agregó la isquemia/ reperfusión placentaria y un probable efecto mecánico de la eyección de sangre que ingresa al espacio intervelloso desde arterias de calibre reducido ${ }^{83,84}$. Recientemente se ha determinado un rol de la decidualización ${ }^{85}$, apoyando un defecto del precondicionamiento endometrial en el embarazo adolescente ${ }^{57}$. (Figura 1).
Fetales: El menor flujo placentario reduce el aporte de oxígeno y nutrientes y en el feto activan el sistema renina-angiotensina, causan remodelación vascular, excesiva producción de especies reactivas de oxígeno que causan mayor compromiso de la función placentaria, liberación de factores placentarios al torrente sanguíneo materno y al compartimento fetal ${ }^{86}$. La adaptación hemodinámica fetal se visualiza mediante el eco Doppler de la arteria umbilical y la cerebral media, anteceden al deterioro del perfil biofísico fetal ${ }^{87}$ y prioriza la perfusión cerebral (brain sparing effect).

\section{Objetivo primario de la vigilancia}

El principal objetivo de vigilar a largo plazo a las mujeres que presentaron embarazos hipertensivos es preve- 
Tabla 3

Cuestionario para evaluar historia obstétrica previa y riesgos cardiovasculares de mujeres que se evalúan preconcepcionalmente o en su primer control obstétrico.

\begin{tabular}{|c|c|c|c|}
\hline \multicolumn{4}{|l|}{ Total de embarazos. $n$} \\
\hline Embarazos espontáneos & No & & \\
\hline Fertilización asistida & Tipo & & \\
\hline Cambio de pareja & No & & \\
\hline \multirow{2}{*}{\multicolumn{4}{|c|}{$\begin{array}{l}\text { Periodo de cohabitación } \\
\text { Abortos espontáneos. n }\end{array}$}} \\
\hline & & & \\
\hline Embarazos hipertensivos & No & ¿Qué embarazo(s)? & Trimestre o semana de gestación \\
\hline Preeclampsia & No & ¿Qué embarazo(s)? & Trimestre o semana de gestación \\
\hline Parto prematuro $<37$ sem & No & ¿Qué embarazo(s)? & Semana de gestación \\
\hline Peso recién nacido <2500 g & No & ¿Qué embarazo(s)? & \\
\hline Diabetes gestacional & No & ¿Qué embarazo(s)? & \\
\hline Hospitalizaciones durante el embarazo. $n$ & No & ¿Qué embarazo(s)? & Causa \\
\hline Otras complicaciones & & ¿Qué embarazo(s)? & \\
\hline Eclampsia, HELLP, & & & \\
\hline mortinatos, DPNI, placenta previa, colestasia. & & & \\
\hline \multicolumn{4}{|l|}{ Edad actual de los hijos (años) } \\
\hline \multicolumn{4}{|l|}{ Sexo de los hijos. M/F } \\
\hline \multicolumn{4}{|l|}{ Riesgos personales } \\
\hline Estado nutricional & Peso & Altura & BMI \\
\hline Tabaquismo & $\mathrm{Si}$ & No & Edad de inicio/suspensión \\
\hline Apnea de sueño & $\mathrm{Si}$ & No & \\
\hline Hipertensión & $\mathrm{Si}$ & No & Edad de inicio tratamiento \\
\hline \multirow{3}{*}{$\begin{array}{l}\text { Hiperlipidemia } \\
\text { Tipo } \\
\text { Ultimo Valor(es) }\end{array}$} & \multirow[t]{3}{*}{$\mathrm{Si}$} & \multirow[t]{3}{*}{ No } & Edad de diagnóstico/tratamiento \\
\hline & & & Colesterol/Triglicéridos/HDL \\
\hline & & & \\
\hline Diabetes & $\mathrm{Si}$ & No & \\
\hline
\end{tabular}

\begin{tabular}{|l|l|l|l|}
\hline Historia familiar & Padre & Madre & Hermanos \\
\hline Causa de muerte (edad) & & & \\
\hline Hipertensión & & & \\
\hline Diabetes & & & \\
\hline Enfermedad coronaria (años) & & \\
\hline Accidente cerebrovascular (años) & & \\
\hline Preeclampsia en madre y hermanas & & \\
\hline que han tenido embarazos & & \\
\hline
\end{tabular}

nir la hipertensión futura de la madre y el hijo. A nivel individual la hipertensión arterial esencial no se presenta bruscamente por lo que es clave detectarla en la etapa subrepticia. La elevación progresiva de las cifras tensionales a lo largo de la vida hace perentorio detectar las etapas prehipertensivas cuando existen cambios funcionales reversibles (vasoconstricción y/o disfunción endotelial) ${ }^{88}$ que generan modificaciones morfo- 


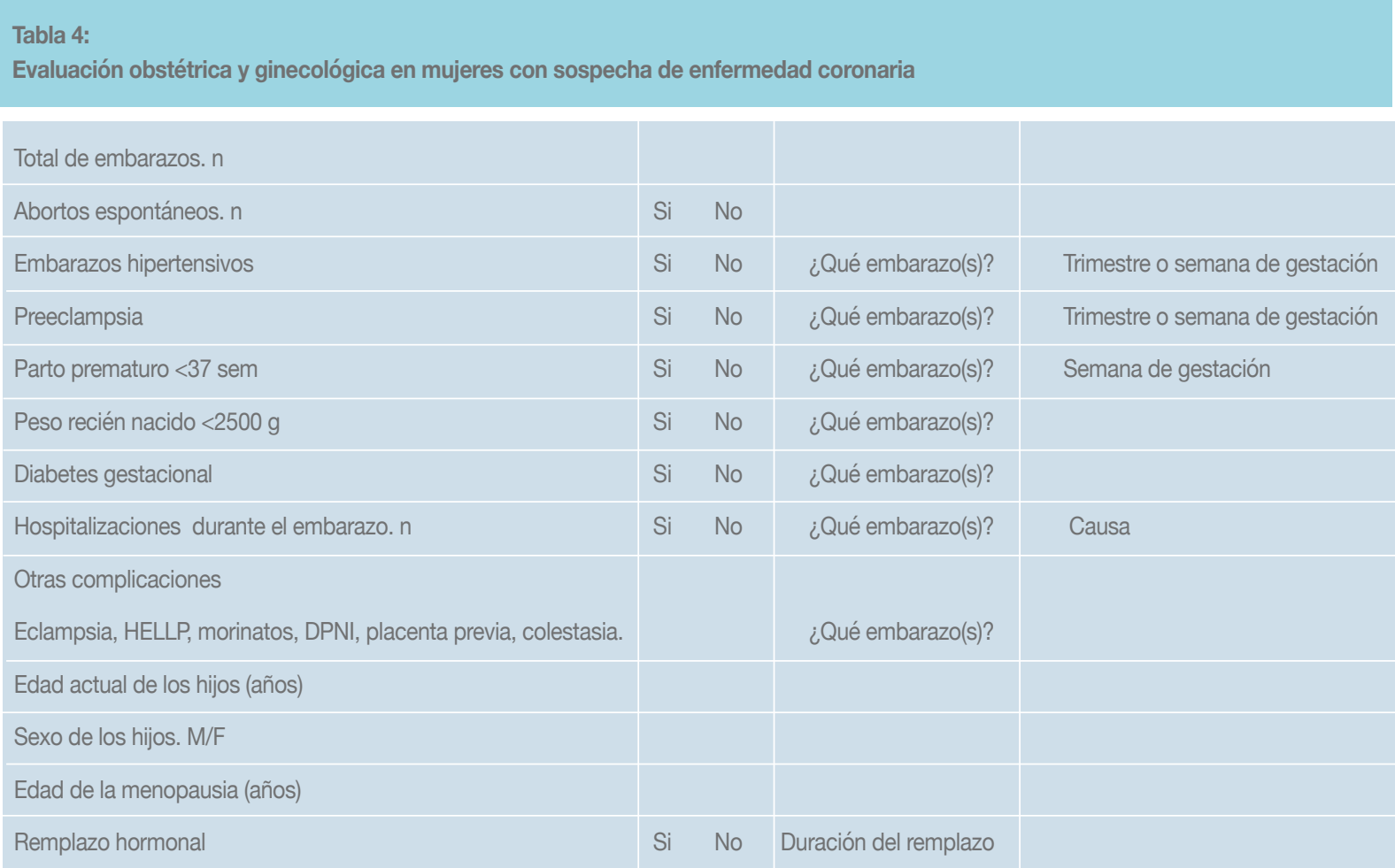

lógicas arteriales (fibrosis, hipertrofia, calcificación, placas ateromatosas) que requieren intervenciones cada vez más enérgicas para reducir las cifras tensionales, las complicaciones y el daño de órganos blancos. En la práctica clínica observamos que existe una etapa de hipertensión lábil/ocasional ante situaciones de stress (ej: "hipertensión de delantal blanco"). Luego las elevaciones tensionales se hacen más frecuentes hasta que sobrepasan el límite de la normotensión. Los diferentes niveles fijados para definir hipertensión establecida entre las últimas guías estadounidenses ${ }^{89}$ y las europeas $^{90}$ (130/80 versus $140 / 90 \mathrm{~mm} \mathrm{Hg}$, respectivamente) refuerzan el continuo de las cifras tensionales y las dificultades para fijar un límite, aunque en ambas guías el corte está basado en evidencias.

El SHE es un factor que desenmascara una hipertensión no diagnosticada y una "bola de cristal" para predecir la hipertensión arterial futura. Tenemos la ventaja que en el país prácticamente todas las madres reciben atención profesional en el parto $(99.7 \% \text { en el } 2016)^{2}$. Si calculamos que entre 8 a $10 \%$ de los embarazos presentan hipertensión 1850 a 2310 de las madres de los 231096 nacidos vivos en el 2016 hubieran sido objeto de seguimiento y recomendaciones preventivas ${ }^{91}$. Además, se deben considerar a las aproximadamente 2000 mujeres que presentaron mortinatos (tasa 8.6 x 1000 nacidos vivos), cuyas causas más importantes son los embarazos hipertensivos, las anomalías congénitas y las infecciones ${ }^{92}$.

\section{Propuesta}

Esta revisión plantea agregar el manejo pre y postconcepcional para enfatizar a los clínicos un continuo que ofrece varias etapas para detección y prevención de embarazos posteriores y de ECV. (Figura 1), para que la prevención cardiovascular se inicie en la práctica obstétrica habitual. También para incorporar la historia obstétrica cuando se sospecha un síndrome coronario. En base al cuestionario de la Global Pregnancy Collaboration (CoLab) ${ }^{97}$ he elaborado borradores para las atenciones obstétricas y cardiológicas, que pueden adaptarse a las prácticas clínicas locales (Tablas 3 y 4). Estos incorporan la necesidad de identificar mujeres con historias obstétricas impecables en el grupo control (sin partos prematuros, desnutrición intrauterina ni abortos espontáneos) y de precisar la suplementación hormonal, dato ausente en estudios que incluyen muje- 


\section{Tabla 5:}

Recomendaciones de la Sociedad Internacional para el Estudio de la Hipertensión en el Embarazo para las mujeres que han presentado embarazos hipertensivos.

Ud. ha presentado un embarazo complicado por hipertensión arterial por lo que nos interesa entregarle información basada en estudios científicos que enfatizan la necesidad de incorporar cuidados que se traducirán en una mejor salud en el mediano y largo plazo.

-Ud. tiene mayores riesgos de enfermedad cardiovascular. muerte. accidente vascular encefálico. diabetes. enfermedad tromboembólica o renal que quienes han tenido embarazos con presión normal.

-Si Ud. presentó preeclampsia tienen un riesgo aproximado de $15 \%$ de repetirla. además de un $15 \%$ de desarrollar hipertensión gestacional y debe recibir aspirina en embarazos futuros. A su vez Ud. deben informar a sus hermanas que tienen un riesgo aumentado de presentar preeclampsia.

-Ud. tiene mayor riesgo de tener hijos pequeños para la edad gestacional en un próximo embarazo aun cuando no presente una PE

- Le aconsejamos que tenga un seguimiento regular por un médico general para controlar la presión arterial y determinar periódicamente grasas y azúcar en sangre.

-Ud. debe advertir a los pediatras si sus hijos han sido prematuros y/o han tenido bajo peso de nacimiento para que sean sometidos a la misma vigilancia.

-Se sabe que si su recién nacido tuvo bajo peso no lo sobrealimente para que alcance el peso normal rápidamente. Al pasar de un ambiente intrauterino que lo hizo "ahorrador" a uno de "abundancia" es más perjudicial que si gana peso lentamente.

- Le recomendamos a Ud. y a su núcleo familiar estilos de vida saludables que incluyan ejercicio aeróbico regular para mantener el peso ideal

Referencia 4. La traducción, adaptación y algunos agregados han sido realizados por GV.

res mayores de 60 años $1^{4,15,98}$.

Es fundamental involucrar a las mujeres que han presentado SHE y convertirlas en protagonistas de su cuidado posterior y en agentes multiplicadores de prevención, por lo que he adaptado las recomendaciones de la Sociedad Internacional para el Estudio de la Hipertensión en el Embarazo $^{4}$. (Tabla 5).

Desde el punto de vista de la investigación biomédica, la iniciativa CoLab proveerá una sólida base para crear registros clínicos y biobancos que contribuirán a dilucidar mediante biología molecular los eslabones de la asociación de enfermedades obstétricas y cardiológicas ${ }^{98}$. Pero en estos momentos esperar el resultado de estos estudios constituye un dilema ético. La evidencia demuestra que ha llegado el tiempo de reconocer tempranamente el riesgo gestacional y remoto de PE.

\section{Agradecimientos}

Agradezco al obstetra Alfredo Germain por incluirme en los primeros estudios que visualizaron la función endotelial y ver en tiempo real la vasodilatación normal o reducida y las curvas del flujo uterino. Al nefrólogo Carlos Zúñiga por recomendarme argumentar con mayor fuerza, como lo ha realizado él para la insuficiencia renal. Por último, a todas las embarazadas hipertensas que he atendido por su fortaleza para proteger al futuro hijo. 


\section{Referencias}

1. GBD 2015 MATERNAL MORTALITY COLLABORATORS. Global, regional and national levels of maternal mortality, 1990-2015: a systematic analysis for the Global Burden of Disease Study. Lancet. 2016; 388: 1775-1812.

2. GONZÁlEZ R, REQUEJO JH, NIEN JK, MERIALDI M, BUSTREO F, BETRAN A. Tackling health inequities in Chile: Maternal, newborn, infant and child mortality between 1990 and 2004. Am J Pub Health. 2009: 99: 1220 -1225.

3. ARABIN B, BASCHAT AA. Pregnancy: An underutilized window of opportunity to improve long-term maternal and infant health - An appeal for continuous family care and interdisciplinary communication. Frontiers in Pediatrics. 2017; 5: 69.

4. BROWN MA, MAGEE LA, KENNY LC, KARUMANCHI SA, MCCARTHY FP, SAITO S, et al. Hypertensive Disorders of Pregnancy: ISSHP Classification, Diagnosis, and Management Recommendations for International Practice. Hypertension. 2018; 72: 24-43.

5. Encuesta Nacional de Salud Chile 2009-2010. Disponible en https://www.minsal.cl/portal/url/item/bcb03d7bc28b64dfe040010165012d23. (consultado el 26 abril 2019).

6. JONSDOTTIR LS, ARNGRIMSSON R, GEIRSSON RT, SIVALDASON H, SIGFUSSON N. Death rates for ischemic heart disease in women with a history of hypertension in pregnancy. Acta Obstet Gynecol Scand. 1995; 74: 772-776.

7. HANNAFORD P, FERRY S, HIRSH S. Cardiovascular sequelae of toxaemia of pregnancy. Heart. 1997; 77: 154-158.

8. SMITH GC, PELL JP, WALSH D. Pregnancy complications and maternal risk of ischaemic heart disease: a retrospective cohort study on 129,290 births. Lancet. 2001; 357: 2002-2006.

9. IRGENS HU, REISAETER L, IRGENS LM, LIE RT. Long term mortality of mothers and fathers after preeclampsia; population based cohort study. BMJ. 2001; 323: 1213-1217.

10. SATTAR N, GREER IA. Pregnancy complications and maternal cardiovascular risk: opportunities for intervention and screening? BMJ. 2002; 325: 157-160.

11. WILSON BJ, WATSON MS, PRESCOTT GJ, et al. Hypertensive diseases of pregnancy and risk of hypertension and stroke in later life: results from cohort study. BMJ. 2003; 326: 845849.

12. PELL JP, SMITH GC, WALSH D. Pregnancy complications and subsequent maternal cerebrovascular events: a retrospecti- ve cohort study of 119,668 births. Am J Epidemiology. 2004; 159: $336-342$.

13. ARNADOTTIR GA, GEIRSSON RT, ARNGRIMSSON R, JONSDOTTIR LS, OLAFSSON O. Cardiovascular death in women who had hypertension in pregnancy: a case-control study. BJOG. 2005; 112: 286-292.

14. CATOV JM, NEWMAN AB, ROBERTS , KELSEY SF, SUTTON-TYRRELL K, HARRIS TB, et al. Preterm delivery and later maternal cardiovascular disease risk. Epidemiology. 2007; 18: 733-739.

15. CRAICI IM, WAGNER SJ, HAYMAN SR, GAROVIC VD. Pre-eclamptic pregnancies: an opportunity to identify women at risk for future cardiovascular disease. Women's Health. 2008; 4: 133-145.

16. CIRILLO PM, COHN BA. Pregnancy complications and cardiovascular disease death: Fifty year follow-up of the Child Health and Development Studies Pregnancy Cohort. Circulation. 2015;132:1234-1242.

17. DEROO L, SKJÆRVEN R, WILCOX A, KLUNGS $\varnothing$ YR K, WIKSTRÖM AK, MORKEN NH, et al. Placental abruption and long-term maternal cardiovascular disease mortality: a population-based registry study in Norway and Sweden. Eur J Epidemiol. 2016; 31:501-511.

18. GRANDI SM, FILION KB, YOON S, AYELE HT, DOYLE CM, HUTCHEON JA, et al. Cardiovascular disease-related morbidity and mortality in women with a history of pregnancy complications. Circulation. 2019;139:1069-1079.

19. ANANTH CV, HANSEN AV, WILLIAMS MA, NYBO ANDERSEN AM. Cardiovascular disease in relation to placental abruption: a population-based cohort study from Denmark. Paediatr Perinat Epidemiol. 2017; 31: 209-218.

20. RIISE HK, SULO G, TELL GS, IGLAND J, NYGÅRD O, VOLLSET SE, et al. Incident coronary heart disease after preeclampsia: Role of reduced fetal growth, preterm delivery, and parity. J Am Heart Assoc 2017;6:e04158.

21. BROUWERS L, VAN DER MEIDEN-VAN ROEST AJ, SAVELKOUL C, VOGELVANG TE, LELY AT, FRANX A, VAN RIJN BB. Recurrence of pre-eclampsia and the risk of future hypertension and cardiovascular disease: a systematic review and meta-analysis. BJOG. 2018; 125: 1642-1654.

22. WHITE WM, MIELKE MM, ARAOZ PA, LAHR BD, BAILEY KR, JAYACHANDRAN M, et al. A history of preeclamp- 
sia is associated with a risk for coronary calcification 3 years later. Am J Obstet Gynecol. 2016; 214: 519.e1-8.

23. VALDÉS G, QUEZADA F, MARCHANT E, VON SCHULTZENDORFF A, MORÁN S, PADILLA O, et al. Association of remote hypertension in pregnancy with coronary artery disease: a case-control study. Hypertension. 2009; 53: 733-738.

24. BELLAMY L, CASAS JP, HINGORANI AD, WILLIAMS DJ. Pre-eclampsia and risk of cardiovascular disease and cancer in later life: systematic review and meta-analysis. BMJ. 2007; 33 : 974-986.

25. MCDONALD SD, MALINOWSKI A, ZHOU Q, YUSUF S, DEVEREAUX PJ. Cardiovascular sequelae of preeclampsia/ eclampsia: a systematic review and meta-analyses. Am Heart J. 2008; 156: 918-930.

26. BROWN MC, BEST KE, PEARSE MS, WAUGH J, ROBSON SC, BELL R. Cardiovascular disease risk in women with pre-eclampsia; systematic review and meta-analysis. Eur J Epidemiol. 2013: 28: 1-19.

27. WU P, HATHTHOTUWA R, KWOK CS, BABU A, KOTRONIAS RA, RUSHTON C, et al. Preeclampsia and future cardiovascular health. A systematic review and meta-analysis. Cardiovasc Qual Outcome. 2017; 10: e003497.

28. CUSIMANO MC, PUDWELL J, RODDY M, CHO CK, SMITH GN. The maternal health clinic: an initiative for cardiovascular risk identification in women with pregnancy-related complications. Am J Obstet Gynecol. 2014; 210: 438.e1-9.

29. SIA WW, MONTGOMERY-FAJIC E, GERMAINE D, WILKIE J, KHURANA R, MARNOCH C, ET al. The postpartum preeclampsia clinic (PPPEC) - an interdisciplinary clinic for cardiovascular risk reduction for women with preeclampsia. Pregnancy Hypertens. 2012; 2: 237.

30. MOSCA L, BENJAMIN EJ, BERRA K, BEZANSON JL, DOLOR RJ, LLOYD-JONES DM, et al. Effectiveness-based guidelines for the prevention of cardiovascular disease in women-2011 update. A guideline from the American Heart Association. J Am Coll Cardiol. 2011; 57: 1404-1423.

31. BUSHNELL C, MCCULLOUGH LD, AWAD IA, CHIREAU MV, FEDDER WN, FURIE KL, et al. Guidelines for the prevention of stroke in women: a statement for healthcare professionals from the American Heart Association/American Stroke Association. Stroke. 2014; 45: 1545-1548.

32. PIEPOLI MF, HOES AW, AGEWALL S, LBUS C, BROTONS C, CATAPANO AL, et al. 2016 European guidelines on cardiovascular disease prevention in clinical practice: The Sixth Joint Task Force of the European Society of Cardiology and other societies on cardiovascular disease prevention in clinical practice Developed with the special contribution of the European Association for Cardiovascular Prevention \& Rehabilitation (EACPR). Eur Heart J. 2016; 37: 2315-2381

33. BAIREY MERTZ CN. Sex, death and the diagnosis gap. Circulation 2014; 130: 740-742.

34. PEPINE CJ, FERDINAND KC, SHAW LJ, LIGHT-MCGROARY KA, SHAH RU, GULATI M, et al. Emergence of nonobstructive coronary artery disease: A woman's problem and need for change in definition on angiography. J Am Coll Cardiol. 2015; 66: 1918-1933.

35. PEPINE CJ, ANDERSON RD, SHARAF BL, REIS SE, SMITH KM, HANDBERG EM, et al. Coronary microvascular reactivity to adenosine predicts adverse outcome in women evaluated for suspected ischemia: results from the NHLBI Women's Ischemia Syndrome Evaluation (WISE). J Am Coll Cardiol. 2010; 55: 2825-2832.

36. Epidemiología de las muertes prematuras en Chile en la década 2001-2010. Disponible en https://www.paho.org/hq/dmdocuments/2014/CHILE-PERFIL-ECV-2014/ (consultado el 21 marzo 2019).

37. BARKER DJ, OSMOND C, GOLDING J, KUH D, WADSWORTH ME. Growth in utero, blood pressure in childhood and adult life, and mortality from cardiovascular disease. BMJ. 1989; 298: 564-567.

38. BARKER DJ. The developmental origins of adult disease. $\mathbf{J}$ Am Coll Nutr. 2004; 23: 588S-S95SS.

39. CALKINS K, DEVASKAR SU. Fetal origins of adult disease. Curr Probl Pediatr Adolesc Health Care. 2011; 41: 158-176.

40. DAVIES EF, LAZDAM M, LEWANDOWSKI AJ, WORTON SA, KELLY B, KENWORTHY Y, el al. Cardiovascular risk factors in children and young adults born to preeclamptic pregnancies: a systematic review. Pediatrics. 2012; 129: e15521561 .

41. LAZDAM M, DAVIES EF, LEWANDOVSKI AJ, WORTON SA, KENWORTHY Y, KELLY B, et al. Prevention of vascular dysfunction after preeclampsia: A potential long-term outcome measure and emerging goal of treatment. J Pregnancy. 2012; 2012: 704146 .

42. FRASER A, NELSON SM, MACDONALD-WALLIS C, SATTAR N, LAWLOR DA. Hypertensive disorders of pregnancy and cardiometabolic health in adolescent offspring. Hypertension. 2013; 62: 614-620.

43. KRAUSE BJ, CASTRO-RODRÍGUEZ JA, UAUY P, CASA- 
NELLO P. Conceptos generales de epigenética: proyecciones en pediatría. Rev Chil Pediatria 2016; 87:4-10.

44. HOVI P, VOHR B, MENT LR. Blood pressure in young adults born at very low birth weight: Adults Born Preterm International Collaboration. Hypertension. 2016; 68: 880-887.

45. DE JONG F, MONUTEAUX MC, VAN ELBURG RM, GILLMAN MW, BELFORT MB. Systematic review and meta-analysis of preterm birth and later systolic blood pressure. Hypertension. 2012; 59: 226-234.

46. GBD 2015 CHILD MORTALITY COLLABORATORS. Global, regional, national, and selected subnational levels of stillbirths, neonatal, infant, and under-5 mortality, 1980-2015: a systematic analysis for the Global Burden of Disease Study 2015. Lancet. 2016; 388: 1725-1774.

47. INE Anuario de Estadísticas Vitales 2014. Disponible en (https://www.ine.cl/docs/default-source/publicaciones/2016/ anuario-de-estad\%C3\%ADsticas-vitales-2014.pdf?sfvrsn=18/ (consultado el 21 abril 2019).

48. DUCKITT K, HARRINGTON D. Risk factors for pre-eclampsia at antenatal booking: systematic review of controlled studies. BMJ. 2005; 33: 565.

49. BERENDS AL, DE GROOT CJ, SIJBRANDS EJ, SIE MP, BENNEHEIJ SH, PAL R, et al. Shared constitutional risks for maternal vascular-related pregnancy complications and future cardiovascular disease. Hypertension. 2008; 51:1034-1041.

50. LOKE YK, BROWN WL, KWOK CS, NIRUBAN A, MYNT PK. Association of obstructive sleep apnea with risk of serious cardiovascular events. A systematic review and meta-analysis. Circ Cardiovasc Qual Outcomes. 2012; 5: 720-728.

51. BIN YS, CISTULLI PA, FORD JB. Population-based sleep apnea in pregnancy and maternal and infant outcomes. J Clin Sleep Med. 2016; 12: 871-877.

52. JOHNSON MP1, BRENNECKE SP, EAST CE DYER TD, ROTEN LT, PROFFITT JM, et al. Genetic dissection of the pre-eclampsia susceptibility locus on chromosome 2q22 reveals shared novel risk factors for cardiovascular disease. Mol Hum Reprod. 2013; 19: 423-437.

53. COLLABORATIVE LOW-DOSE ASPIRIN STUDY IN PREGNANCY COLLABORATIVE GROUP. CLASP: a randomised trial of low-dose aspirin for the prevention and treatment of pre-eclampsia among 9364 pregnant women. Lancet. 1994; 343: 619-629.

54. BUJOLD E, ROBERGE S, LACASSE Y, BUREAU M, AUDIBERT F, MARCOUX S, et al. Prevention of preeclampsia and intrauterine growth restriction with aspirin started in early pregnancy: a meta-analysis. Obstet Gynecol. 2010; 116: 402414.

55. ANTITHROMBOTIC TRAILISTS (ATT) COLLABORATION. Aspirin in the primary and secondary prevention of vascular disease: collaborative meta-analyisi of individual participant data from randomised trial. Lancet. 2009; 373: 18491860.

56. STAFF AC, DECHEND R, PIJNENBORG R. Learning from the placenta acute atherosis and vascular remodeling in preeclampsia-Novel aspects for atherosclerosis and future cardiovascular health. Hypertension. 2010; 56: 1026-1034.

57. BROSENS I, BENAGIANO G, BROSENS JJ. The potential perinatal origin of placentation disorders in the young primigravida. Am J Obstet Gynecol. 2015; 212: 580-585.

58. JEYABALAN A, POWERS RW, DURICA AR, HARGER GF, ROBERTS JM, NESS RB. Cigarette smoke exposure and angiogenic factors in pregnancy and preeclampsia. Am J Hypertens. 2008 ; 21: 943-947.

59. CELERMAJER D. Endothelial dysfunction: Does it matter? Is it reversible? J Amer Coll Cardiol. 1997; 30: 325-333.

60. IRRIBARRA V, GERMAIN AM, CUEVAS A, FAÚNDEZ L, VALDÉS G. Disfunción endotelial como alteración primaria en las patologías vasculares Rev Méd Chile. 2000; 128: 659670 .

61. CRAVEN CM, MORGAN T, WARD K. Decidual spiral artery remodelling begins before cellular interaction with cytotrophoblasts. Placenta 1988; 19:241-252.

62. KAUFMANN P, BLACK S, HUPPERTZ B. Endovascular trophoblast invasion: implications for the pathogenesis of intrauterine retardation and preeclampsia. Biol Reprod. 2003; 69: $1-7$.

63. PARRA M, RODRIGO R, BARJA P, BOSCO C, FERNÁNDEZ V, MUÑOZ H, SOTO-CHACÓN E. Screening test for preeclampsia through assessment of uteroplacental blood flow and biochemical markers of oxidative stress and endothelial dysfunction. Am J Obstet Gynecol. 2005; 193: 1486-1491.

64. GERMAIN AM, ROMANIK MC, GUERRA I, SOLARI S, REYES MS, JOHNSON RJ. et al. Endothelial dysfunction: a link among preeclampsia, recurrent pregnancy loss, and future cardiovascular events? Hypertension 2007; 49:90-95.

65. ROBERTS JM, TAYLOR RN, MUSCI TJ, RODGERS GM, HUBEL CA, MCLAUGHLIN MK. Preeclampsia: an endothelial cell disorder. Am J Obstet Gynecol. 1998; 161: 1200-1204. 
66. REDMAN CW, SACKS GP, SARGENT IL. Preeclampsia: an excessive maternal inflammatory response to pregnancy. Am J Obstet Gynecol. 1999; 180: 499-506.

67. LEVINE RJ, MAYNARD SE, QIAN C, LIM KH, ENGLAND $\mathrm{LJ}, \mathrm{YU} \mathrm{KF}$, et al. Circulating angiogenic factors and the risk of preeclampsia. N Engl J Med. 2004; 350: 672-683.

68. EGELAND GM, KLUNGSOYR K, OYEN N, TELL GS, NAESS O, SKJAERVEN R. Preconceptional cardiovascular risk factor differences between gestational hypertension and preeclampsia. Hypertension. 2016; 67: 1173-1180

69. HARVILLE EW, VIIKARI JSA, RAITAKKKARI OL. Preconceptional cardiovascular risk factors and pregnancy outcome. Epidemiology. 2011; 22: 724-730.

70. HEDDERSON MM, DARBINIAN JA, SRIDHAR SB, QUESENBERRY CP. Prepregnacy risk cardiometabolic and inflammatory risk factors and subsequent risk of hypertensive disorders of pregnancy. Am J Obstet Gynecol. 2012; 207: 68.e1-68.e9

71. MAGNUSSEN EB, VATTEN LJ, LUND-NIELSEN TI, SALVESEN KA, SMITH GD, ROMUNDSTAD PR. Prepregnancy cardiovascular risk factors as predictive of pre-eclampsia: population based cohort. BMJ. 2007; 335: 978.

72. VALDÉS G, KAUFMANN P, CORTHORN J, ERICES R, BROSNIHAN KB, JOYNER-GRANTHAM J. Vasodilator factors in the systemic and local adaptations to pregnancy. Reprod Biol Endocrinol. 2009; 7: 79.

73. VALDÉS G, CORTHORN J. Challenges posed to the maternal circulation by pregnancy. Integr Blood Press Control. 2011; 4: 45-53.

74. WILLIAMS D. Pregnancy: a stress test for life. Curr Opin Obstet Gynecol. 2003; 15: 465-471.

75. WEISSGERBER TL, MILIC NM, MILIN-LAZOVIC JS, GAROVIC V. Impaired flow-mediated dilation before, during and after preeclampsia: A systematic review and metanalysis. Hypertension. 2016; 67: 415-423.

76. HENRIQUES ACPT, CARVALHO FHC, FEITOSA HN, MACENA RHM, MOTA RMS, ALENCAR JCG. Endothelial dysfunction after pregnancy-induced hypertension. Int J Obstet Gynecol. 2014; 124: 230-234.

77. AYKAS F, SOLAK Y, ERDEN A, BULUT K, DOGAN S, SARLI B, ET AL. Persistence of cardiovascular risk factors in women with previous preeclampsia: a long-term follow-up study. J Investig Med. 2015; 63: 641-645.
78. ÖSTLUND E, AL-NASHI M, HAMAD RR, LARSSON A, ERIKSSON M, BREMME K, ET AL. Normalized endothelial function but sustained cardiovascular risk profile 11 years following a pregnancy complicated by preeclampsia. Hypertens Res. 2013; 36: 1081-1087.

79. SANDVIK MK, LEIRGUL E, NYGÅRD O, UELAND PM, BERG A, SVARSTAD E, VIKSE BE. Preeclampsia in healthy women and endothelial dysfunction 10 years later. Am J Obstet Gynecol. 2013; 209: 569.e1-569.e10

80. GRAND`MAISON S, PILOTE L, OKANO M, LANDRY T, DAYAN N. Markers of vascular dysfunction after hypertensive disorders of pregnancy. A systematic review and meta-analysis. Hypertension. 2016; 68: 1447-1458.

81. MILIC NM, MILIN-LAZOVIC J, WEISSGERBER TL, TRAJKOVIC G, WHITE WM, GAROVIC VD. Preclinical atherosclerosis at the time of pre-eclamptic pregnancy and up to 10 years postpartum: systematic review and meta-analysis. Ultrasound Obstet Gynecol. 2017; 49: 110-115.

82. JUONALA M, VIIKARI JS, LAITINEN T, MARNIEMI J, HELENIUS H, RÖNNEMAA T, RAITAKARI OT. Interrelations between brachial endothelial function and carotid intima-media thickness in young adults: the cardiovascular risk in young Finns study. Circulation. 2004; 110: 2918-2923.

83. HUNG T-H, SKEPPER JN, CHARNOCK-JONES DS, BURTON GJ. Hypoxia/reoxygenation: A potent inducer of apoptotic changes in the human placenta and possible etiological factor in preeclampsia. Circ Res. 2002; 90: 1274-1281.

84. BURTON GJ, WOODS AW, JAUNIAUX E, KINGDOM JCP. Rheological and physiological consequences of conversion of the maternal spiral arteries for uteroplacental blood flow during human pregnancy. Placent. 2009; 30: 473-482.

85. RABAGLINO MB, POST UITERWEER ED, JEYABALAN A, HOGGE WA, CONRAD KP. Bioinformatics approach reveals evidence for impaired endometrial maturation before and during early pregnancy in women who developed preeclampsia. Hypertension. 2015; 65: 421-429.

86. ALJUNAIDY MM, MORTON JS, COOKE CLM, DAVIDGE ST. Prenatal hypoxia and placental oxidative stress: Linkages to developmental origins of cardiovascular disease. Am J Physiol Regul Integr Comp Physiol. 2017; 313: R395-R399.

87. BASCHAT A, GEMBRUCH U, HARMAN CR. The sequence of changes in Doppler and biophysical parameters as severe fetal growth restriction worsens. Ultrasound Obstet Gynecol. 2011; 18: 571-577.

88. BONETTI PO, LERMAN LO, LERMAN A. Endothelial dys- 
function a marker of atherosclerotic risk.. Arterioscler Thromb Vasc Biol. 2003; 23: 168-175.

89. JAMES PA, OPARIL S, CARTER BL, CUSHMAN WC, DENNISON-HIMMELFARB C, HANDLER J, et al. 2014 evidence-based guideline for the management of high blood pressure in adults: Report from the panel members appointed to the Eight Joint National Committee (JNC 8). JAMA. 2014; 311: 507520 .

90. WILLIAMS B, MANCIA G, SPIERING W, AGABITI ROSEI E, AZIZI M, BURNIER M, et al. 2018 ESC/ESH Guidelines for the management of arterial hypertension. Eur Heart J. 2018;39:3021-3104.

91. Serie Nacimientos 2000-2015. Disponible en http://www.deis. cl/wp-content/uploads/2011/05/SerieNacimientos_2000_2015/ (consultado 15 abril 2019).

92. OVALLE A, FUENTES A, CHACÓN V, ESPINOZA C, GONZÁLEZ R, RAMOS M, et al. Método de clasificación de mortinatos según condición obstétrica relevante de la muerte fetal, en un hospital público de Chile (Método CORM). Rev Med Chile. 2016; 144: 1020-1028.

93. VILLAR J, ABDEL-ALEEM H, MERIALDI M, MATHAI M, ALI MM, ZAVALETA N, et al. World Health Organization randomized trial of calcium supplementation among low calcium intake pregnant women. Am J Obstet Gynecol. 2006; 194: 639-649.
94. VADILLO-ORTEGA F, PERICHART-PERERA O, ESPINO S, AVILA-VERGARA MA, IBARRA I, AHUED R, et al. Effect of supplementation during pregnancy with L-arginine and antioxidant vitamins in medical food on pre-eclampsia in high risk population: randomised controlled trial. BMJ. 2011; 342: d2901.

95. GERMAIN AM, VALDÉS G, ROMANIK MC, REYES MS. Evidence supporting a beneficial role for long-term L-arginine supplementation in high-risk pregnancies. Hypertension. 2004; 44: e1.

96. COSTANTINE MM, CLEARY K, HEBERT MF, AHMED MS, BROWN LM, REN Z, et al. Safety and pharmacokinetics of pravastatin used for the prevention of preeclampsia in high-risk pregnant women: a pilot randomized controlled trial. Am J Obstet Gynecol. 2016; 214:720.e1.

97. STAFF AC, REDMAN CWG, WILLIAMS D, LEESON P, MOE K, THILAGANATHAN B, ET al. Pregnancy and longterm maternal cardiovascular health: Progress through harmonization of research cohorts and biobanks. Hypertension. 2016; 67: 251-261.

98. BAIREY MERZ CN, SHUFELT C, JOHNSON BD, AZZIZ R, BRAUNSTEIN GD. Reproductive hormone exposure timing and ischemic heart disease: Complicated answers to a simple question. Maturitas. 2010; 65: 297-298. 